\title{
ANTIMONY(III) COMPLEXES \\ OF 2-(N-SALICYLIDENE)-5-CHLOROBENZOPHENONE: SYNTHESIS, SPECTROSCOPIC STUDIES AND REACTIVITY WITH RESPECT TO SULFUR DONOR LIGANDS
}

\author{
M. S. Singh* and K. Prasada Rao \\ Organic \& Organometallic Chemistry Laboratory, School of Studies in Chemistry, \\ Vikram University, Ujjain (M.P.) 456 010, India
}

\begin{abstract}
Dichloro, chlorobis and tris [2-( $\mathrm{N}$-salicylidene)-5-chlorobenzophenone] derivatives of antimony(III) have been synthesised by the reactions of antimony trichloride with the sodium salt of 2-( $\mathrm{N}$-salicylidene)-5-chlorobenzophenone (prepared insitu) in 1:1, 1:2 and 1:3 molar ratios, respectively The substitution reactions of these newly synthesised chloro derivatives have been studied with alkyl xanthates. All these compounds are characterised by elemental analyses, molecular vieight measurements and spectroscopic (IR, ${ }^{1} \mathrm{H}$ and ${ }^{13} \mathrm{C}$ NMR) studies.
\end{abstract}

\section{INTRODUCTION}

Complexes of antimony containing oxygen and sulfur donor ligands have been $i$.... $\mathrm{n}$ for many years ${ }^{12}$, and are found to be biologically active ${ }^{3}$ and carcinostatic ${ }^{4}$. A few antimony compuunds are known for their technical uses in catalytic processes ${ }^{5.6}$. The metal complexes derived from 2-( $\mathrm{N}$ salicylidene)-5-chlorobenzophenone are reported to act as potentlal antianxiety ${ }^{8}$ and antiepileptlc agents. In a recent study the, $\mathrm{Cu}(\mathrm{II})$ and $\mathrm{Zn}$ (II) complexes of this moiety have been shown to possess antiinflammatory ${ }^{10}$ activity also. Persual of above literature and in continuation of our earlier work ${ }^{11} 1 \%$. we have undertaken a systematic investigation on these and $r(t)$ lort here isynthesis, characterisation and structural features of a few complexes of antimony(III) and their substitution reactions.

\section{RESULTS AND DISCUSSION}

Dichloro, chlorobis and tris-antimony(III) derivatives of $2-(\mathrm{N}$-salicylidene)-5chlorobenzophenone are synthesised by the reaction of antimony trichloride with the sodium salt of the ligand in $1: 1,1: 2$ and $1: 3$ molar ratios, respectively.

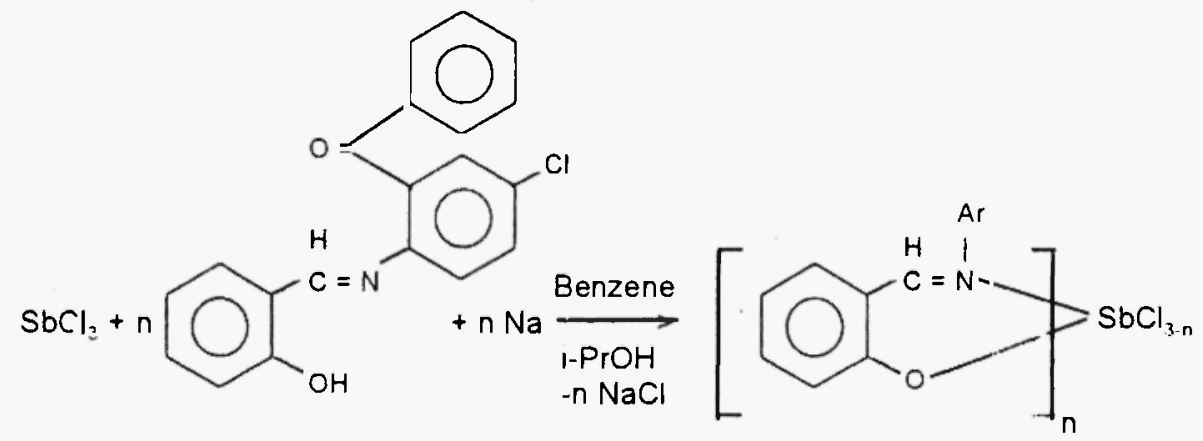

Where $n=1$, Compd (I); $n=2$, Compd. (II); $n=3$, Compd. (III).

These derivatives were prepared by refluxing the reactants in benzene for about $4-5$ hours 
to ensure the completion of the reaction. The desired product could be isolated by evaporation of the solvent under reduced pressure, after filtering off the precipitated sodium chloride. These were further purified by crystallisation from benzene-petroleum ether $\left(40-60^{\circ}\right)$ mixtures. The mixed derivatives are synthesised by the reaction of dichloro (I) and chlorobis(II) compounds with potassium alkyldithiocarbonates in carbon disulfide benzene in 1:2 and 1:1 molar ratios, respectively.

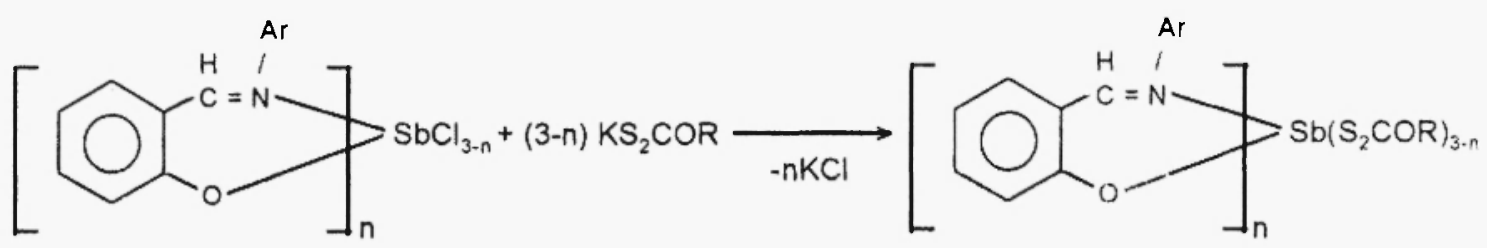

Where $n=2, R=$ Me, Compd. (1); $n=1, R=M e$, Compd. (2); $n=2, R=E t$, Compd. (3); $n=1, R$ $=$ Et, Compd. (4); $n=2, R=i-P r$, Compd. (5); $n=1, R=i-P r$, Compd. (6); $n=2, R=n-B u$, Compd. (7); $n=1, R=n-B u$, Compd. (8); $n=2, R=i-B u$, Compd. (9); $n=1, R=i-B u$, Compd. (10).<smiles>Cc1ccc(Cl)cc1Oc1ccccc1</smiles>

The mixed derivatives were synthesised by stirring the reactants in dry carbon disulfide/ benzene for about 3 hours. The $\mathrm{KCl}$ was filtered off and the compound was isolated and purified by crystallisatior. from benzene/carbon disulfied mixtures. All these complexes are yellow crystalline solids, soluble in common organic solvents and are monomeric in benzene

\section{IR spectral data}

The infrared spectra of these complexes have been recorded on a Perkin-Elmer $580 \mathrm{~B}$ spectrometer, using $\mathrm{KBr}$ pellets. The spectrum of the ligand shows bands in the regions $3380-3260$ $\mathrm{cm}^{-1}, 1680 \mathrm{~cm}^{-1}$ and $1620 \mathrm{~cm}^{-1}$ which have been assigned to $v(\mathrm{OH}), v(C=O)$ and $v(C=N)$ respectively ${ }^{13}$. The disappearance of $\mathrm{v}(\mathrm{OH})$ in the complexes indicate deprotonation of phenolic $-\mathrm{OH}$ and consequent coordination to antimony, which is further substantiated by the appearance of new bands $i$ region $465-445 \mathrm{~cm}^{-1}$ and $350 \mathrm{~cm}^{-1}$ assignable to $v(\mathrm{Sb}-\mathrm{O})$ and $v(\mathrm{Sb}-\mathrm{Cl})$ respectively. "The bai. I present at $1620 \mathrm{~cm}^{-1}$ due to $v(\mathrm{C}=\mathrm{N})$ is found to be shifted for about $20-25 \mathrm{~cm}^{-1}$ to lower wave number in the complexes suggesting coordination of $C=N$ to antimony. $A$ band at $1680 \mathrm{~cm}^{-1}$ in the ligand, which can be assigned to keto carbonyl does not show any discernible shift in the spectra of the complexes suggesting the non-participation of the keto group in coordination.

Infrared spectra of all the mixed ligand complexes of antimony(III) show bands in the regions $1030-1005 \mathrm{~cm}^{-1}$ and $330-290 \mathrm{~cm}^{-1}$ due to $v(\mathrm{C}=\mathrm{S})$ and $v(\mathrm{Sb}-\mathrm{S})$ vibrations respectively ${ }^{14}{ }^{15}$. The presence of a strong band in the region $1030-1005 \mathrm{~cm}^{-1}$ due to $v(C=S)$ indicates bidentate nature of the xanthate moiety ${ }^{1 \epsilon}$.

\section{NMR spectral data}

The ' $H$ NMR spectrum of the ligand is characterised by appearance of a signal at 012.40 ppm for phenolic - OH group, at $\delta 10.60 \mathrm{ppm}$ for azomethine group and a multiplet at $\delta 6.84-7.86$ $\mathrm{ppm}$ attributable to protons of phenyl moiety. The resonance at $\delta 12.40 \mathrm{ppm}$ is absent in the spectra 
of the complexes suggesting deprotonation of phenolic - $\mathrm{OH}$ and its subsequent involvement in coordination. The coordination through $\mathrm{C}=\mathrm{N}$ is supported by the down field shift of the free ligand signal at $\delta 10.60 \mathrm{ppm}$ to $\delta 11.55 \mathrm{ppm}$ in the complexes ${ }^{17}$. The resonance due to phenyl moiety remains uneffected. The xanthate moities also exhibited the expected proton resonances ${ }^{14}$ is due to their respective alkoxy protons.

${ }^{13} \mathrm{C}$ NMR spectra of these derivatives have been recorded. The aromatic carbon resonances of these derivatives were asigned by using incremental rules for aromatic substitution. The number of ' $\mathrm{C}$ NMR signals correspond to the number of chemically different carbon atoms present in these compounds. There is no significant chemical shift for the various ${ }^{13} \mathrm{C}$ signals present in the mix $\equiv d$ ligand complexes of antimony(III) as compared to the corresponding tris (alkyldithiocarbonate) antimony(III) and tris [2-( $\mathrm{N}$-(salicylidene)-5-chlorobenzophenone] antimony(III) complexes.

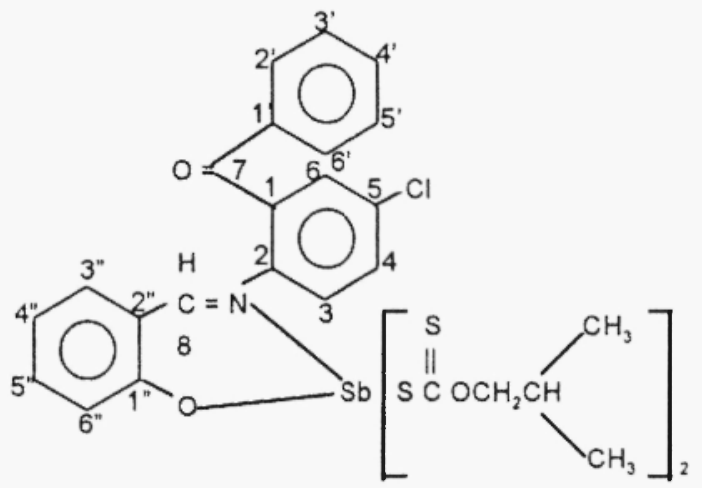

Thus based on the elemental analysis, molecular weight measurements and spectroscopic studies a bidentate mode of attachment of the both types of ligands to the metal atom is proposed. The dichloro derivative possess trigonal bipyramidal geometry with the lone pair in equatorial site(a), and bis derivative has octahedral geometry with the lone pair occupying an axial position(b) while tris derivative and mixed derivatives show a distorted octahedral geometry if the presence of the stereochemically active lone pair is also considered in the capping position (c, d, e) with pseudo seven coordinated structures.<smiles></smiles>

(a)

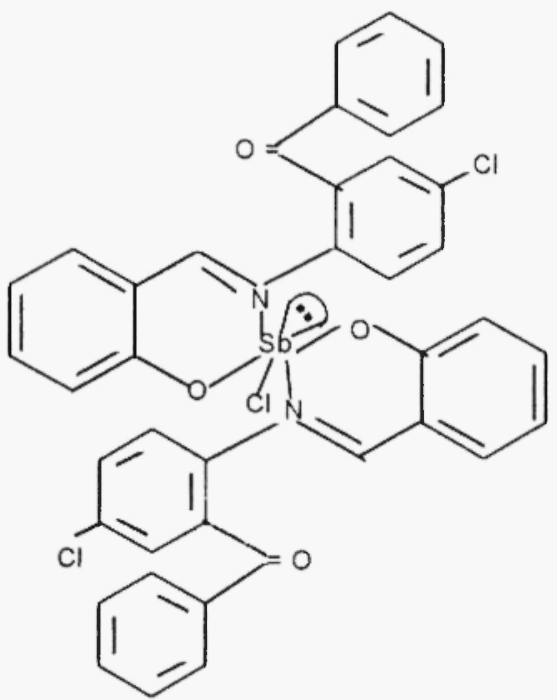

(b) 


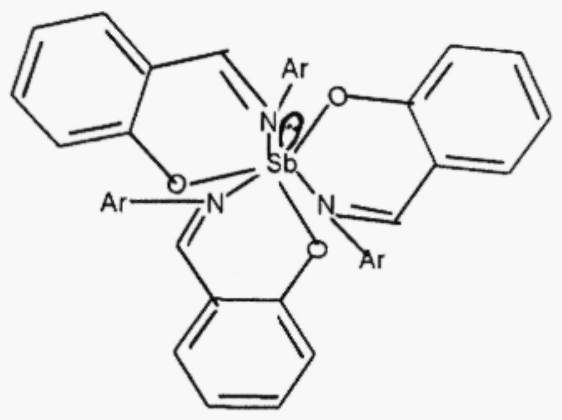

(c)

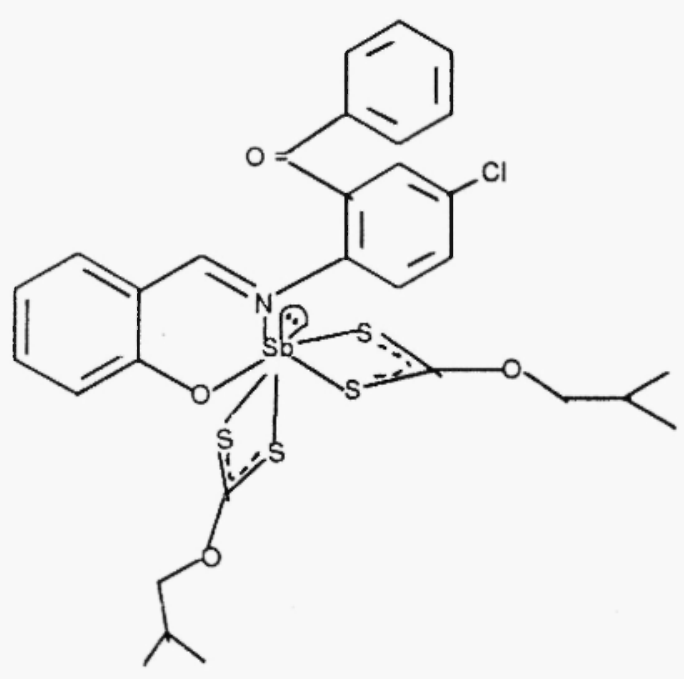

(d)

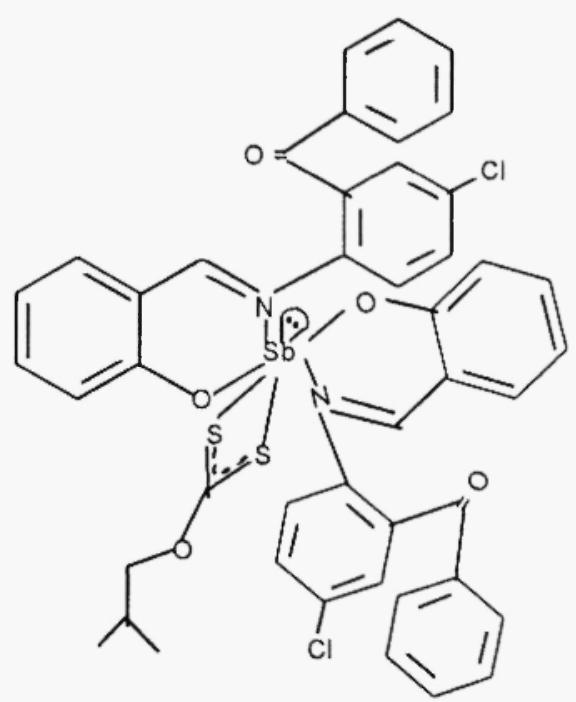

(e)

\section{EXPERIMENTAL}

All reactions and manipulations were performed under an inert atmosphere. All solvents were freshly distilled over appropriated drying agents immediately prior to use ${ }^{18}$. All the chemicals used were of reagent grade. Antimony trichloride was distilled under reduced pressure before use. 2-( $\mathrm{N}$-(salicylidene)-5-chlorobenzophenone ${ }^{10}$ and potassium alkylxanthates ${ }^{19}$ were prepared using a previously described procedure. Antimony was estimated iodometrically ${ }^{20}$ after converting it to antimony $(V)$; sulfur was estimated gravimetrically as barium sulphate ${ }^{21}$ and nitrogen was $\epsilon \quad$ rlated by Kjeldahl's method ${ }^{22}$. The IR spectra were recorded on a Perkin-Elmer $580 \mathrm{~B}$ instrument. The 'H NMR spectra were recorded on a Perkin-Elmer R-32 and ${ }^{13} \mathrm{C}$ NMR spectra, on a JEOL FX-90, using TMS as an external standard.

Reaction between $\mathrm{SbCl}_{3}$ and the sodium salt of 2-(N-salicylidene)-5-chlorobenzophenone in a $1: 1$ molar ratio :

To an excess of isopropanol-benzene mixture $(20 \mathrm{ml})$ a weighed quantity of $\mathrm{Na}$ metal $(0.14 \mathrm{~g}$. $6 \mathrm{~m}$ mole) was added and refluxed for about half an hour till a clear solution of the sodium isopropoxide was obtained. After cooling $2.01 \mathrm{~g}(6 \mathrm{mmole})$ of 2-( $\mathrm{N}$-salicylidene $)$-5-chlorobenzophenone and about 
$15 \mathrm{ml}$ of benzene was added and the mixture was refluxed for 2 hours on an oil bath. After cooling, $1.36 \mathrm{~g}(6 \mathrm{mmole})$ of $\mathrm{SbCl}_{3}$ was added and the mixture was further refluxed for 2-3 hours to ensure the completion of the reaction. The desired product was isolated after filtering off the precipitated $\mathrm{NaCl}$. The product was further purified by crystallisation from benzene-petroleum ether $\left(40-60^{\circ} \mathrm{C}\right)$ mixture.

All the other antimony(III) derivatives of 2-( $\mathrm{N}$-salicylidene)-5-chlorobenzophenone were synthesised similarly.

Reaction between Chlorobis [2-(N-salicylidene)-5-chlorobenzophenone] antimony(III) and potassium methyl dithiocarbonate in a 1:1 molar ratio :

Potassium methyl dithiocarbonate and chlorobis [2-(N-salicylidene)-5-chlorobenzophenone] antimony(III) were stirred in benzene-carbon disulfide mixture (20 ml each) in 1:1 molar ratio for $\sim 3$ hours at room temperature. The precipitated $\mathrm{KCl}$ was removed by filtration, and the desired compound could be obtained by removing the solvent under reduced pressure. The compound was further purified by crystallisation from benzene-carbondisulfide mixture.

The other mixed derivatives were synthesised similarly. The characterisation of the compounds is given below.

Compound I: $\mathrm{C}_{20} \mathrm{H}_{13} \mathrm{NO}_{2} \mathrm{SbCl}_{2}$ :

Yield, 96\%; Mol. Wt. [F(C)] : $525(527.55)$ yellow solid; M.P., $129-130^{\circ} \mathrm{C}$.

Analysis [\%F(C)] : Sb, 20.75(23.07); Cl, $18.13(20.18) ; \mathrm{N} 2.42(2.65)$

IR $\left(\mathrm{cm}^{-1}\right): v(\mathrm{C}=\mathrm{O}), 1660 ; v(\mathrm{C}=\mathrm{N}), 1610 ; v(\mathrm{Sb}-\mathrm{O}), 510$

PMR (jppm) : 6.65-7.60, m, 12H (Ph); 3.60, s, $1 \mathrm{H}(\mathrm{CH})$

${ }^{13} \mathrm{C}$ NMR ( $\left.\delta \mathrm{ppm}\right): 197.4, \mathrm{C}-8 ; 164.30, \mathrm{C}-7 ; 134.11, \mathrm{C}-1 ; 135.12, \mathrm{C}-2 ; 138.0, \mathrm{C}-3 ; 129.15, \mathrm{C}-4$; 133.09, C-5; 129.0, C-6; 135.50, C-1'; 116.15, C-2'; 129.01, C-3'; 118.90, C-4'; 128.10, C-5'; 117.50 C-6'; 151.56, C-1"; 137.00, C-2"; 132.11, C-3"; 127.19, C-4"; 127.95, C-5"; 130.59, C-6".

Compound II : $\left[\mathrm{C}_{20} \mathrm{H}_{13} \mathrm{NO}_{2}\right]_{2} \mathrm{SbCl}$ :

Yield. $94 \%$; Mol. Wt. [F(C)] : $830(826.85)$ yellow solid; M.P., $135-137^{\circ} \mathrm{C}$

Analysis $[\% \mathrm{~F}(\mathrm{C})]$ : $\mathrm{Sb}, 14.78(14.72) ; \mathrm{Cl}, 11.51(12.88) ; \mathrm{N} 3.22(3.38)$

IR $\left(\mathrm{cm}^{-1}\right): v(\mathrm{C}=\mathrm{O}), 1655 ; v(\mathrm{C}=\mathrm{N}), 1615 ; v(\mathrm{Sb}-\mathrm{O}), 515$

PMR (cippm) : 6.60-7.62, m, 24H (Ph); 3.70, s, 2H (CH)

${ }^{13} \mathrm{C}$ NMR $(\delta \mathrm{ppm}): 196.4, \mathrm{C}-8 ; 165.30, \mathrm{C}-7 ; 134.22, \mathrm{C}-1 ; 136.12, \mathrm{C}-2 ; 138.9, \mathrm{C}-3 ; 130.15, \mathrm{C}-4$ 133.29, C-5; 130.0, C-6; 136.50, C-1'; 116.55, C-2'; 129.31, C-3'; 118.90, C-4'; 128.40, C-5'; 116.50 C-6'; 150.56, C-1"; 137.40, C-2"; 132.61, C-3"; 126.79, C-4"; 127.95, C-5"; 130.59, C-6".

Compound III : $\left[\mathrm{C}_{20} \mathrm{H}_{13} \mathrm{NO}_{2}\right]_{3} \mathrm{Sb}:$

Yield, 97\%; Mol. Wt. [F(C)] : $1127(1125.15)$ yellow solid; M.P., $136-138^{\circ} \mathrm{C}$

Analysis [\% F(C)] : Sb, $10.32(10.82) ; \mathrm{N} 3.61(3.73)$

IR $\left(\mathrm{cm}^{-1}\right): v(C=0), 1650 ; v(C=N), 1610 ; v(\mathrm{Sb}-\mathrm{O}), 520$

PMR (ippm) : 6.80-7.85, $\mathrm{m}, 36 \mathrm{H}(\mathrm{Ph}) ; 3.85, \mathrm{~s}, 3 \mathrm{H}(\mathrm{CH})$

${ }^{13} \mathrm{C}$ NMR (o ppm) : 195.01, C-8; 163.74, C-7; 135.54, C-1; 136.32, C-2; 137.3, C-3; 129.54, C-4; 132.43, C-5; 128.65, C-6; 135.1, C-1'; 116.80, C-2'; 128.15, C-3'; 119.74, C-4'; 128.65, C-5'; 117.8, C-6'; 153.1, C-1"; 136.52, C-2"; 131.13, C-3"; 127.95, C-4"; 128.09, C-5"; 131.13, C-6".

Compound 1 : $\left[\mathrm{C}_{20} \mathrm{H}_{13} \mathrm{NO}_{2}\right] \mathrm{Sb}\left(\mathrm{S}_{2} \mathrm{COCH}_{3}\right)_{2}$ :

Yield, $96 \%$; Mol. Wt. [F(C)] : $672(670.55)$ yellow solid; M.P., $114-116^{\circ} \mathrm{C}$.

Analysis [\%F(C)] : Sb, 17.35 (18.16); S, $18.00(19.09) ; N 1.99(2.08)$

IR $\left(\mathrm{cm}^{-1}\right) ; v(C=O), 1640 ; v(C=N), 1590 ; v(\mathrm{Sb}-\mathrm{S}), 320 ; v(\mathrm{C}=\mathrm{S}), 1020$

PMR (oppm) : 6.83-7.78, m, 12H (Ph); 3.25, s, $1 \mathrm{H}(\mathrm{CH}) ; 0.98, \mathrm{~s}, 6 \mathrm{H}\left(\mathrm{CH}_{3}\right)$

${ }^{1} \mathrm{C}$ NMR (o ppm) : 196.98, C-8; 163.44, C-7; 135.54, C-1; 134.94, C-2; 138.10, C-3; 129.15, C-4; 
133.19, C-5; 128.55, C-6; 135.50, C-1'; 116.75, C-2'; 129.76, C-3'; 118.10, C-4'; 128.09, C-5'; 117.3, C-6"; 151.56, C-1"; 137.0, C-2"; 132.17, C-3"; 127.95, C-4"; 130.59, C-5"; 130.02, C-6"; 190.16, $\mathrm{C}=\mathrm{S} ; 15.60,-\mathrm{OCH}_{3}$.

\section{Compound $2:\left[\mathrm{C}_{20} \mathrm{H}_{13} \mathrm{NO}_{2}\right]_{2} \mathrm{Sb} \mathrm{S}_{2} \mathrm{COCH}_{3}$ :}

Yield, 91\%; Mol. Wt. [F(C)] : 888 (898.35) yellow solid; M.P. $119-121^{\circ} \mathrm{C}$

Analysis [\% $\mathrm{F}(\mathrm{C})]$ : Sb, 11.05 (13.55); S, 7.97 (7.12); N 2.97 (3.11)

IR $\left(\mathrm{cm}^{-1}\right): v(\mathrm{C}=\mathrm{O}), 1635 ; v(\mathrm{C}=\mathrm{N}), 1590 ; v(\mathrm{Sb}-\mathrm{S}), 315 ; v(\mathrm{C}=\mathrm{S}), 1020$

PMR (ippm) : 6.90-7.85, m, 24H (Ph); 3.20, s, 2H (CH); $1.05, \mathrm{~s}, 3 \mathrm{H}\left(\mathrm{CH}_{3}\right)$

${ }^{1: C}$ NMR (i ppm) : 197.3, C-8; 162.12, C-7; 132.19, C-1; 135.04, C-2; 138.06, C-3; 129.9, C-4; 133.02, C-5; 128.05, C-6; 133.60, C-1'; 117.8, C-2'; 130.0, C-3'; 117.11, C-4'; 130.09, C-5'; 119.3, C6'; 151.4, C-1"; 138.14, C-2"; 133.7, C-3"; 127.6, C-4"; 128.34, C-5"; 130.02, C-6"; 190.66, -C=S; 15.98, $-\mathrm{OCH}_{3}$.

Compound $3:\left[\mathrm{C}_{20} \mathrm{H}_{13} \mathrm{NO}_{2}\right] \mathrm{Sb}\left(\mathrm{S}_{2} \mathrm{COC}_{2} \mathrm{H}_{8}\right)_{2}$ :

Yield, $91 \%$; Mol. Wt. $[\mathrm{F}(\mathrm{C})]: 678(698.55)$ yellow solid; M.P., $116-117^{\circ} \mathrm{C}$.

Analysis [\% $\mathrm{F}(\mathrm{C})]$ : Sb, $17.12(17.43) ; \mathrm{S}, 17.58(18.32) ; \mathrm{N} 2.01(2.00)$

IR $\left(\mathrm{cm}^{-1}\right): v(\mathrm{C}=\mathrm{O}), 1635 ; v(\mathrm{C}=\mathrm{N}), 1595 ; v(\mathrm{Sb}-\mathrm{S}), 315 ; v(\mathrm{C}=\mathrm{S}), 1015$

PMR (cppm) : 6.90-7.80, m, 12H (Ph); 3.20, s, $1 \mathrm{H}(\mathrm{CH}) ; 1.25, \mathrm{t}, 6 \mathrm{H}\left(\mathrm{CH}_{3}\right) ; 4.40, \mathrm{q}, 4 \mathrm{H}\left(\mathrm{OCH}_{2}\right)$

${ }^{13} \mathrm{C}$ NMR (o ppm) : 196.9, C-8; 163.11, C-7; 132.79, C-1; 136.84, C-2; 139.21, C-3; 127.9, C-4; 133.52, C-5; 128.05, C-6; 133.64 , C-1'; 117.52, C-2'; 129.92, C-3'; 117.84, C-4'; 129.00, C-5'; 119.76 , C-6"; 152.54, C-1"; 137.00, C-2"; 132.8, C-3"; 128.6, C-4"; 128.0, C-5"; $130.76, C-6 " ; 188.32,-C=S$; $63.4,-\mathrm{CH}_{2} ; 13.01,-\mathrm{CH}_{3}$.

Compound $4:\left[\mathrm{C}_{20} \mathrm{H}_{13} \mathrm{NO}_{2}\right]_{2} \mathrm{Sb} \mathrm{S}_{2} \mathrm{COC}_{2} \mathrm{H}_{6}$ :

Yield, $95 \%$; Mol. Wit. [F(C)] : $915(912.35)$ yellow solid; M.P., $113-115^{\circ} \mathrm{C}$.

Analysis [\% $\mathrm{F}(\mathrm{C})]$ : Sb, 12.55 (13.34); S, 6.91 (7.01); N 2.95 (3.06)

IR $\left(\mathrm{cm}^{-1}\right): v(\mathrm{C}=0), 1650 ; v(\mathrm{C}=\mathrm{N}), 1595 ; v(\mathrm{Sb}-\mathrm{S}), 320 ; v(\mathrm{C}=\mathrm{S}), 1012$

PMR (oppm) : 6.95-7.75, m, 24H (Ph); 3.25, s, 2H (CH); 1.20, t, 3H $\left(\mathrm{CH}_{3}\right), 4.45, \mathrm{q}, 2 \mathrm{H}$ (CCti )

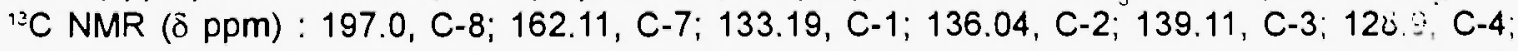
133.02, C-5; 128.95, C-6; 134.64, C-1'; 117.0, C-2'; 129.31, C-3'; 118.1, C-4'; 129.09, C-5'; 119.0, C6'; 152.3, C-1"; 137.12, C-2"; 133.1, C-3"; 128.0, C-4"; 128.34, C-5"; 131.12, C-6"; 189.36, -C=S; $62.5,-\mathrm{CH}_{2} ; 13.41,-\mathrm{CH}_{3}$.

Compound $5:\left[\mathrm{C}_{20} \mathrm{H}_{13} \mathrm{NO}_{2}\right] \mathrm{Sb}\left(\mathrm{S}_{2} \mathrm{COC}_{3} \mathrm{H}_{7}\right)_{2}$ :

Yield, 90\%; Mol. Wt. [F(C)] : 731 (726.55) yellow solid; M.P., $115-117^{\circ} \mathrm{C}$

Analysis $[\% \mathrm{~F}(\mathrm{C})]$ : Sb, $15.88(16.76) ; \mathrm{S}, 17.00(17.62) ; \mathrm{N} 1.77(1.93)$

IR $\left(\mathrm{cm}^{-1}\right): v(C=0), 1636 ; v(C=N), 1605 ; v(S b-S), 310 ; v(C=S), 1015$

PMR (oppm) : 6.95-7.82, m, 12H (Ph); 3.28, s, $1 \mathrm{H}(\mathrm{CH}) ; 1.40, \mathrm{~d}, 12 \mathrm{H}\left(\mathrm{CH}_{3}\right) ; 5.10, \mathrm{~m}, 1 \mathrm{H}(\mathrm{OCH})$

${ }^{13} \mathrm{C}$ NMR $(\delta \mathrm{ppm}): 194.99, \mathrm{C}-8 ; 165.36, \mathrm{C}-7 ; 134.66, \mathrm{C}-1 ; 135.33, \mathrm{C}-2 ; 137.9, \mathrm{C}-3 ; 130.0, \mathrm{C}-4$; $133.55, C-5 ; 130.0, C-6 ; 136.12, C-1^{\prime} ; 117.1, C-2^{\prime} ; 129.35, C-3^{\prime} ; 118.3, C-4^{\prime} ; 127.11, C-5^{\prime} ; 116.95, C-$ 6'; 152.1, C-1"; 137.12, C-2"; 133.11, C-3"; 128.18, C-4"; 127.34, C-5"; 130.26, C-6"; 190.4, C=S; $51.5,-\mathrm{OCH} ; 23.2,-\mathrm{CH}_{3}$.

\section{Compound $6:\left[\mathrm{C}_{20} \mathrm{H}_{13} \mathrm{NO}_{2}\right]_{2} \mathrm{Sb} \mathrm{S}_{2} \mathrm{COC}_{3} \mathrm{H}_{7}$ :}

Yield, 94\%; Mol. Wt. [F(C)] : $921(925.35)$ yellow solid; M.P., $118-121^{\circ} \mathrm{C}$ Analysis $[\% \mathrm{~F}(\mathrm{C})]$ : Sb, $14.82(13.15) ; \mathrm{S}, 5.21(6.91) ; \mathrm{N} 2.88$ (3.02)

IR $\left(\mathrm{cm}^{-1}\right): v(\mathrm{C}=\mathrm{O}), 1660 ; v(\mathrm{C}=\mathrm{N}), 1600 ; v(\mathrm{Sb}-\mathrm{S}), 310 ; v(\mathrm{C}=\mathrm{S}), 1020$

PMR (jppm) : 7.00-7.95, m, 24H (Ph); 3.30, s, 2H (CH); 1.45, d, 6H $\left(\mathrm{CH}_{3}\right), 5.05, \mathrm{~m}, 1 \mathrm{H}(\mathrm{OCH})$

${ }^{13} \mathrm{C}$ NMR $(\delta \mathrm{ppm}): 195.22, \mathrm{C}-8 ; 164.11, \mathrm{C}-7 ; 133.92, \mathrm{C}-1 ; 137.42, \mathrm{C}-2 ; 137.15, \mathrm{C}-3 ; 131.21, \mathrm{C}-4$; 134.31, C-5; 130.12, C-6; 136.5, C-1'; 117.95, C-2'; 130.02, C-3'; 121.12, C-4'; 127.04, C-5': 117.22 , 
C-6'; 151.56, C-1"; 138.16, C-2"; 130.92, C=3"; 127.92, O-4"; 124.42, C.5"; 131.12, C-6"; 190.91, $\mathrm{C}=\mathrm{S} ; 52.0, . \mathrm{OCH} ; 24.5,-\mathrm{CH}_{3}$ :

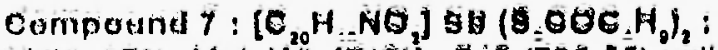

Ylete, 87\%; Mol. Wt. [F (C)] : 7a 2 (738.55) yellow solid; M.P., $111.113^{\circ} \mathrm{C}$

Analysis [\% $\%(C)]: S b, 15.72(16.49) ; S, 16.89(17.33) ; N 1.69(1.89)$

IR $\left(\mathrm{cm}^{-1}\right) ; v(C=0), 1640 ; v(C=N), 1580 ; v(S b-S), 315 ; v(C=S), 1010$

PMR (oppm) : 6.85-7.85, $\mathrm{m}, 12 \mathrm{H}(\mathrm{Ph}) ; 3.15, \mathrm{~s}, 1 \mathrm{H}(\mathrm{CH}) ; 0.90, \mathrm{t}, 6 \mathrm{H}\left(\mathrm{CH}_{3}\right) ; 1.75, \mathrm{~m}, 8 \mathrm{H}\left(\mathrm{CH}_{2}\right) 4.40$, 1. $4 \mathrm{H}\left(\mathrm{OCH}_{2}\right)$

${ }^{12}$ C NMR (i ppm) : 196.72, C-8; 165.81, C-7; 134.92, C-1; 136.92, C-2; 137.15, C-3; 130.11, C-4; 135.41, C-5; 129.12, C-6; 136.5 , C-1'; 117.05, C-2'; 129.12, C-3'; 120.32 , C-4'; 127.74, C-5'; 118.02 , C-6'; 150.36, C-1"; 138.16, C-2"; 130.92, C-3"; 127.02, C-4"; 125.12, C-5"; 132.12, C-6"; 189.34,$\mathrm{C}=\mathrm{S} ; 65.0,-\mathrm{OCH}_{2} ; 23.1,-\mathrm{CH}_{2} ; 12.1,-\mathrm{CH}_{3}$.

Compound $8:\left[\mathrm{C}_{20} \mathrm{H}_{13} \mathrm{NO}_{2}\right]_{2} \mathrm{Sb} \mathrm{S}_{2} \mathrm{COC}_{4} \mathrm{H}_{9}$ :

Yield, $88 \%$; Mol. Wt. [F(C)]: $941(932.35)$ yellow solid; M.P., $114-116^{\circ} \mathrm{C}$.

Analysis $[\% \mathrm{~F}(\mathrm{C})]$ : Sb, $13.45(13.05) ; \mathrm{S}, 5.37(6.86) ; \mathrm{N} 2.92(3.00)$

IR $\left(\mathrm{cm}^{-1}\right): v(C=0), 1653 ; v(C=N), 1605 ; v(S b-S), 300 ; v(C=S), 1010$

PMR (sppm) : $6.80-7.80, \mathrm{~m}, 24 \mathrm{H}(\mathrm{Ph}) ; 3.20, \mathrm{~s}, 2 \mathrm{H}(\mathrm{CH}) ; 0.85, \mathrm{t}, 3 \mathrm{H}\left(\mathrm{CH}_{3}\right), 4.35, \mathrm{t}, 2 \mathrm{H}\left(\mathrm{OCH}_{2}\right) ; 1.80$, m. $4 \mathrm{H}\left(\mathrm{CH}_{2}\right)$

'? NMR (j ppm) : 195.72, C-8; 165.31, C-7; 134.62, C-1; 137.12, C-2; 137.95, C-3; 129.11, C-4; 134.01, C-5; 129.12, C-6; 136.0, C-1'; 117.55, C-2'; 129.72, C-3'; 119.32, C-4'; 127.04, C-5'; 118.42, C.6'; 151.36, C-1"; 138.1, C-2"; 131.82, C-3"; 127.42, C-4"; 126.32, C-5"; 132.12, C-6"; 189.34, $\mathrm{C}=\mathrm{S} ; 65.39,-\mathrm{OCH}_{2} ; 23.6,-\mathrm{CH}_{2} ; 11.5,-\mathrm{CH}_{3}$.

Compound $9:\left[\mathrm{C}_{20} \mathrm{H}_{13} \mathrm{NO}_{2}\right] \mathrm{Sb}\left(\mathrm{S}_{2} \mathrm{COC}_{4} \mathrm{H}_{9}\right)_{2}$ :

Yield, $92 \%$; Mol. Wit. [F(C)]: $731(738,55)$ yellow solid; M.P., $116-118^{\circ} \mathrm{C}$.

Analysis [\% F(C)] : Sb, $16.32(16.49) ; S, 16.42(17.33) ; N 1.71(1.89)$

IR $\left(\mathrm{cm}^{-1}\right) \cdot v(C=O), 1620 ; v(C=N), 1575 ; v(\mathrm{Sb}-\mathrm{S}), 320 ; v(\mathrm{C}=\mathrm{S}), 1012$

PMR (ippm) : 6.95-7.80, m, $12 \mathrm{H}(\mathrm{Ph}) ; 3.30, \mathrm{~s}, 1 \mathrm{H}(\mathrm{CH}) ; 0.95, \mathrm{~d}, 12 \mathrm{H}\left(\mathrm{CH}_{3}\right) ; 1.65, \mathrm{~m}, 4 \mathrm{H}\left(\mathrm{OCH}_{2}\right)$ ${ }^{13} \mathrm{C}$ NMR $(\delta \mathrm{ppm}): 196.50, \mathrm{C}-8 ; 166.30, \mathrm{C} .7 ; 133.9, \mathrm{C}-1 ; 134.9, \mathrm{C}-2 ; 138.51, \mathrm{C}-3 ; 129.7, \mathrm{C}-4$; 132.71, C-5; 129.65, C-6; 135.72, C-1'; 116.75, C-2'; 130.0, C-3'; 117.85, C-4'; 129.10, C-5'; 116.19, C-6'; 154.0, C-1"; 136.90, C-2"; 132.0, C-3"; 129.43, C-4"; 126.51, C-5"; 129.12, C-6"; 191.4, C=S; $67.2,-\mathrm{OCH}_{2} ; 31.2,-\mathrm{CH} ; 23.5,-\mathrm{CH}_{3}$.

Compound $10:\left[\mathrm{C}_{20} \mathrm{H}_{13} \mathrm{NO}_{2}\right]_{2}$ Sb S $\mathrm{COC}_{4} \mathrm{H}_{9}$ :

Yield, $95 \%$; Mol. Wi. [F(C)] : $936(932.35)$ yellow solid; M.P., $121-124^{\circ} \mathrm{C}$

Analysis $[\% \mathrm{~F}(\mathrm{C})]$ : Sb, $12.30(13.05) ; \mathrm{S}, 6.15(6.86) ; \mathrm{N} 2.87(3.00)$

IR $\left(\mathrm{cm}^{-1}\right): v(C=0), 1660 ; v(C=N), 1595 ; v(S b-S), 305 ; v(C=S), 1015$

PMR (ìppm) : 6.65-7.80, m, 24H (Ph); 3.20, s, 2H(CH);0.90, d, 3H( $\left(\mathrm{CH}_{3}\right) ; 1.70, \mathrm{~m}, 2 \mathrm{H}\left(\mathrm{OCH}_{2}\right)$

1:C NMR (i ppm) : 196.70, C-8; 166.50, C-7; 133.0, C-1; 134.5, C-2; 137.51, C-3; 129.7, C-4; 132.11, C-5; 129.15, C-6; 134.72, C-1'; 117.05, C-2'; 130.5, C-3'; 117.85, C-4'; 129.10, C-5'; 117.10, C-6"; $154.5, C-1 " ; 136.95, C-2 " ; 132.5, C-3 " ; 129.43, C-4 " ; 126.51, C-5^{\prime \prime} ; 129.12, C-6 " ; 190.8, C=S$; $68.1,-\mathrm{OCH}_{i} ; 30.9,-\mathrm{CH}_{2} 23.9,-\mathrm{CH}_{3}$.

\section{REFERENCES}

1. S. K. Pandey, R. Hasselbring, A. Steiner, D. Stalke and H.W. Roesky, Polyhedron, 12, 2941 (1993).

2. L.D. Freedman and G.O. Doak, J. Organomet. Chem., 404, 49 (1991).

3. R. Karra, Y.P. Singh and A.K. Rai, Indian J. Chem., 32A, 78 (1993). 
4. M.A. Ali and S.E. Livingstone, Coord. Chem. Rev., 13, 101 (1974).

5. C.A. McAuliffe in Comprehensive Coordination Chemistry, eds. G Wilkinson, R.D. Gillard and J.A. McCleverty, Pergaman, Oxford, 3 (1987).

6. G. Alonzo, n. Bertazzi and M. Consiglio, Inorg. Chim. Acta, 85, 115 (1984).

7. L.H. Sternbach, J. Med. Chem., 22 (1979).

8. J.L. Howard and G.T. Pollard, Drugs of Today, 14, 47 (1978).

9. L. De Angelis, Drugs of today, 15, 107 (1979).

10. I. U. Khan, S.K. Srivastava and S.C. Srivastava, Indian J. Chem., 26A, 238 (1987).

11. R.J. Rao, K. Prasada Rao and M.S. Singh, Natl. Acad. Sci. Lett., 19, 193 (1996).

12. K. Prasada Rao, K.R. Choudhary, M.S. Singh and R.J. Rao, Indian J. Chem., (in press) 1997.

13. K. Nakamoto, Infrared Spectra of Inorganic and Coordination Compounds, Wiley, New York (1970).

14. R. K. Gupta, A.K. Rai and R.C. Mehrotra, Indian J. Chem., 24A, 752 (1985).

15. R. K. Gupta, A.K. Rai, R.C. Mehrotra and V.K. Jain, Polyhedron, 6, 721 (1984).

16. F. Bonati and R. Ugo, J. Organomet. Chem., 10, 257 (1967).

17. N.S. Biradar, G.V. Karajagi, V.L. Roddabasamgoudar and T.M. Aminabhavi, Indian J. Chem., 24A, 620 (1985).

18. B.S. Furniss, A.J. Hammford, P.W.G. Smith and A.R. Tatchell, "Vogel's Text Book of Practical Organic Chemistry", 5th edn., Longman, U.K., (1991).

19. A.I. Vogel Practical Organic Chemistry, Longmans Green, London 644 (1948).

20. B.P. Singh, G. Srivastava and R.C. Mehrotra, J. Organomet. Chem., 171, 35 (1979).

21. H.P.S. Chauhan, G. Srivastava and R.C. Mehrotra, Polyhedron, 2, 359 (1983).

22. J. Basset, R.C. Denney, G.H. Jeffery and J. Mendham, "Vogel's Textbook of Quantitative Inorganic Analysis", 4th edn., Longman, U.K. (1978).

Received: August 8, 1997 - Accepted: September 11, 1997 Accepted in revised camera-ready format: November 26, 1997 